\title{
Quantitative Analysis of Mast Cells in Benign Odontogenic Tumors
}

\author{
Kanupriya Gupta*, Jatin Gupta, Rahul Agrawal and Neha Verma
}

Faculty of Dental Sciences, IMS, BHU, Varanasi (U.P.), 221005, India

\begin{abstract}
The aim of this study was to investigate the presence of mast cells in a series of odontogenic tumors. Fortyfive cases of odontogenic tumors were investigated using $1 \%$ toluidine blue for mast cells, and differences between groups were statistically evaluated. Mast cells were present in $96 \%$ of odontogenic tumors. Mast cells are probably one of the major components of the Stromal scaffold in odontogenic tumors.
\end{abstract}

Keywords: Mast cells, odontogenic tumors, toluidine blue.

\section{INTRODUCTION}

Odontogenic tumors (OTs) are derived from epithelial and/or ectomesenchymal cells of either the forming dental organ or associated structures. They are considered to be important for both oral and maxillofacial surgery and pathology as they cause extensive bone destruction [1].

Due to significant clinical and histopathological diversity between the entities that are part of their spectrum, OTs are heterogeneous and can vary from a non neoplastic proliferation to a benign or malignant lesion with metastatic capacity [2].

Mast cells (MCs) are bone marrow-derived cells that are widely found in human tissues. They are classified according to the secretion of proteins called endopeptidases and are divided into tryptases and chymases [3]. MCs are also considered to be important in tumorigenesis because they play a key role in the dynamics of tumor growth. hese cells influence angiogenesis, tissue remodeling and a host's immunological response [4]. Moreover, there is evidence of an association between the density of MCs and the process of tumor development. These cells are primarily present close to the tumor, interacting with the tumoral microenvironment via surface molecules, cytokines and growth factors, effectively contributing to the progression of the tumor [5].

The tissue stroma plays an essential role in the preservation of epithelial tissues as minor alterations in the epithelial tissue are followed by corresponding changes in the stroma [6]. Given that MCs may accumulate on sites at which neoplastic lesions develop and that little is known about both their role and behavior in OTs, the aim of this study was to

*Address correspondence to this author at the Faculty of Dental Sciences, IMS, BHU, Varanasi (U.P.), 221005, India; Tel: 7976487542;

E-mail: drkanupriya12@gmail.com examine the distribution of MCs present in a series of benign OTs.

\section{MATERIAL AND METHODS}

The present study constituted of 45 histopathologically diagnosed, formalin-fixed, paraffin embedded tissue samples of Odontogenic tumors. Histological diagnosis were revised and classified by an experienced oral pathologist based upon the current World Health Organization (WHO) classification [7].

The sample consisted of 10 solid ameloblastomas (SAs),10 unicystic ameloblastomas (UAs), 10 keratocystic odontogenic tumors, six adenomatoid odontogenic tumors (AOTs), five odontogenic myxomas (OMs), and four cystic calcifying odontogenic tumors (CCOTs).

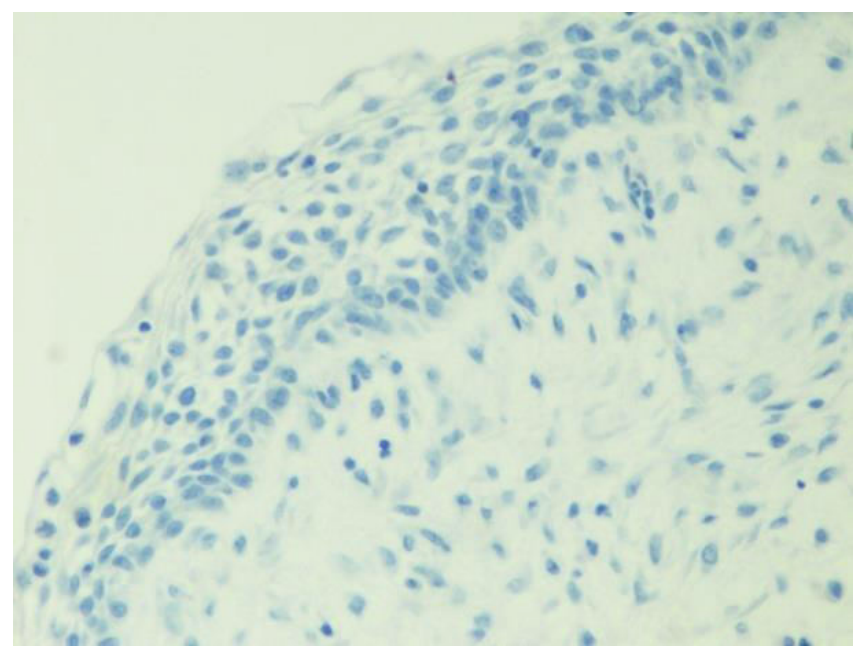

Figure 1: Unicystic ameloblastoma.

\section{Staining For Mast Cells}

The samples were retrieved from archives which were fixed in formalin embedded in paraffin, serially sectioned at 5um thick and stained with hematoxylin and eosin to confirm the diagnosis. Cases which had 
overlapping features were not considered in the study. Later confirmed cases were stained with $1 \%$ toluidine blue to visualize the mastcells. Afterwards the sections were dehydrated, cleared and mounted with DPXmountant.

\section{Quantifying Mast Cells}

Histological analyses included the observation of the MCs on both stroma and the parenchyma of the tumors, avoiding areas of significant inflammation. When the MCs were observed on the parenchyma of the lesion, they were considered to be intraparenchymal. When they were located at the stroma, they were referred to as periparenchymal.

However, the total count including both areas was also considered. In addition, the shape of the cells and the presence of degranulated MCs were also described.

The histomorphometric assessment was carried out by two trained observers using high definition light microscopy $\times 400$ (Axiostar Plus, Zeiss, Germany, $\times$ 400 ) in up to 10 high power fields using a digital camera for recording (Axiocam Icc3, Zeiss, Germany), and both observers were blind to the microscopic diagnosis and previous value of count. Mast cell count per square millimeter was performed using a specific software (Axiovision Rel 4.8, Zeiss, Germany 2008). Quantification of positive cells was calculated by the mean count found in each case.

Differences between groups were evaluated using the Kruskal - Wallis test followed by the Dunn' s test and the Mann - Whitney test. A p -value $<0.05$ was considered to be statistically significant.

\section{RESULTS}

MCs were present in $96 \%$ of tumors, except for two ameloblastoma cases (one solid and one unicystic). Morphologically, these cells had an elongated, oval, and/or rounded shape, commonly located close to blood vessels. In general, MCs were primary located in the periparenchymal region of the OTs, whereas relatively fewer MCs were found within the tumor's parenchyma. A summary of the statistical analysis is seen on Table 1.

MCs present in solid ameloblastoma were observed in the tumor stroma surrounding more solid and follicular epithelial islands, with or without squamous metaplasia. The intraparenchymal MCs were seen at the periphery of the tumor islands, and in areas corresponding to stellate reticulum of ameloblastomas. The OM showed few MCs, which were more frequently located next to the bone trabeculae within the tumor and adjacent to areas of bone sclerosis.

Regarding OMs, MCs were exclusively seen in the extracellular matrix. In this tumor, it is not possible to establish a well-defined boundary between the tumoral stroma and the parenchyma. In OTs with a cystic structure (KOT, UA, CCOT, and AOT), the MCs were distributed throughout all areas of the lesion including the deepest areas of the cystic capsule, the intermediate areas and the areas close to the epithelial lining mainly in KOTs. Those areas next to the epithelial lining showed the highest concentration of cells except when there was subepithelial hyalinization such as in UA. In addition, a few mast cells were located within the cystic epithelium and in areas close to calcification.

Table 1: Distribution of MCs in Benign Odontogenic Tumors

\begin{tabular}{|c|c|c|c|c|}
\hline Tumor & $\begin{array}{l}\text { Periparenchymal } \\
\text { count (Mean } \pm S D \text { ) }\end{array}$ & $\begin{array}{l}\text { Intraparenchymal } \\
\text { count (Mean } \pm S D \text { ) }\end{array}$ & $\begin{array}{l}\text { Total count } \\
\text { (Mean } \pm S D)\end{array}$ & $\begin{array}{l}\text { Periparenchymalx } \\
\text { intraparenchymal }\end{array}$ \\
\hline Solid ameloblastoma & $2.63 \pm 1.64$ & $0.49 \pm 0.63$ & $3.02 \pm 0.63$ & $p<0.05^{a}$ \\
\hline Unicystic ameloblastoma $^{b}$ & $2.51 \pm 1.16$ & $0.67 \pm 0.82$ & $3.16 \pm 1.43$ & $p<0.05^{a}$ \\
\hline $\begin{array}{l}\text { Keratocystic odontogenic } \\
\text { tumor }^{b}\end{array}$ & $2.66 \pm 1.33$ & $0.46 \pm 0.21$ & $2.82 \pm 1.82$ & $p<0.05^{a}$ \\
\hline Odontogenic myxoma & - & - & $1.30 \pm 0.26$ & - \\
\hline $\begin{array}{l}\text { Adenomatoid odontogenic } \\
\text { adenomatoid }^{\mathrm{b}}\end{array}$ & $0.95 \pm 0.28$ & $0.80 \pm 0.77$ & $1.75 \pm 0.62$ & $p>0.05^{a}$ \\
\hline $\begin{array}{l}\text { Calcifying cystic odontogenic } \\
\text { tumor }^{\mathrm{b}}\end{array}$ & $1.95 \pm 0.79$ & $2.25 \pm 1.78$ & $2.76 \pm 0.66$ & - \\
\hline
\end{tabular}

${ }^{a}$ Mann -Whitney test.

${ }^{\mathrm{b}} \mathrm{In}$ these lesions, the periparenchymal region corresponded to the fibrous cystic wall whereas the intraparenchymal area corresponded to the epithelial lining 


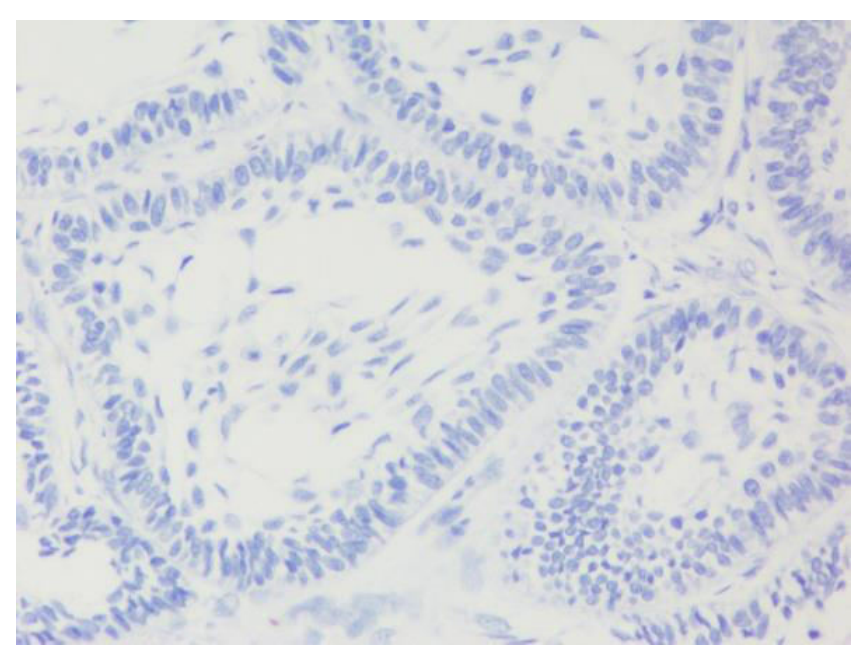

Figure 2: Solid Ameloblastoma.

Another finding observed was the presence of an intense degranulation process of mast cells in the vicinity of bone tissue such as those tumors presenting bone sclerosis and amidst bone trabeculae except for AOT that showed an absence of MCs in those areas. Degranulated MCs were also surrounding or amidst ghost cells as well as close to and in areas of inflammation. Further, MCs were located in and near the epithelial parenchyma of AOTs.

Using the Kruskal - Wallis test, a statistical comparison of the total density of MCs between all OTs showed no significant difference $(p>0.05)$. However, a significant difference was found when the UA and KOT were compared to the OM using the Dunn' $s$ test ( $p$ $<0.05$ ) as both UA and KOT exhibited greater MCs density. Similarly, using the Mann - Whitney test, there was also no statistically significant difference when comparing the density of intraparenchymal and periparenchymal MCs. However, a greater MCs distribution was found in KOT in relation to $A O(p$ $<0.01$ ).

In general, the presence of MCs in different types of OTs was weakly associated with a significant inflammatory response as no case of inflammation was detected in $24(51 \%)$ of the 45 tumors. Further, of the 23 cases that showed some typical histological sign of acute or chronic inflammation, 19 were considered to be nonsignificant. Regarding all odontogenic tumors, significant inflammation was only observed in two cases of KOT cases.

\section{DISCUSSION}

Tumor development represents dynamic processes, which are regulated by molecular changes induced by tumor cells themselves as well as by favorable microenvironmental conditions that provide essential "tools" for survival and cell multiplication including certain cytokines, growth factors and cellular components such as MCs [5]. Due to these functions, we believe that the MCs may also play an important role in the growth and expansion of OTs.

In the present study, MCs were found in $96 \%$ of the OTs analyzed. Depending upon their location and stage of maturation, MCs express different quantities of surface antigens that are involved in cell activation and recognition [7]. Further, the secretion of endopeptidases by MCs modulates inflammation, matrix destruction, tissue remodeling, and angiogenesis [8].

We found that the MCs were most frequently located in the periparenchymal region, whereas relatively fewer were observed within the tumor parenchyma. According to Maltby et al. [9], the location of MCs in periparenchymal regions of tumors suggests that the recruitment of these cells might occur due to their migration from healthy surrounding tissues or from the migration of progenitors of MCs via blood vessels close to the tumor.

Regarding cystic odontogenic neoplasms, we found a greater concentration of MCs in the subepithelial region of the cystic fibrous wall when compared with deeper or more distant areas in relation to the cystic lining. These findings might be due to the greater accumulation of heparin in the extracellular matrix found in this region, which could serve as a source of proangiogenic substances through the secretion of vascular endothelial growth factor (VEGF) and fibroblast growth factor-2 (FGF2) [10]. In addition, we also observed the presence of MCs within the cystic lining. It is possible that the matrix proteins and fibrogenic cytokines secreted by cystic odontogenic epithelium have a chemotactic effect on mast cells and facilitate their accumulation [11]. Furthermore, degranulated MCs surrounding ghost cells within the OCCT cystic lining might indicate tumor rejection based on the enzyme content present in MCs.

In this study, although we avoided areas of inflammation, we did not observe an association between the quantity of MCs and the presence of a significant inflammatory process. These results corroborate the findings of Smith et al. [10] who also found no relationship between the presence of these cells and inflammation present in KOTs. 
We did not find a statistically significant difference between all OTs and MCs. However, it is important to state that there is a clear association between the accumulation of MCs and the processes of tumor growth and progression, which are usually associated with poor prognosis [12].

Solid ameloblastoma is considered to be more aggressive than UA. However, the results of this study did not show a significant distinction for MCs, considering the biological behavior of both lesions. We did not observe significant differences in the density of total MCs in both solid and UA even when the intraparenchymal and peritumoral areas were considered separately.

$\mathrm{OM}$ is a benign and invasive neoplasia, with a high potential for bone destruction and recurrence [1]. In this study, this tumor showed a smaller amount of MCs when compared to the tumors with similar clinical behavior such as ameloblastoma and KOT. Nevertheless, this difference was only significant when compared with the KOT. Although other parameters should be studied to contribute to the data about the biological behavior of OM, this study showed that there was a significant difference in MC count between UA and $\mathrm{OM}$.

In this study, the AOT displayed a low number of MCs. This is a noninvasive benign tumor that is less aggressive. Moreover, the absence of MCs in the areas of bone sclerosis could explain the nonaggressive behavior of this lesion as there would be an insufficient amount of proteolytic enzymes capable of destroying the extracellular matrix involved.

Despite the low number of CCOT cases, we also observed a concentration of MCs in the epithelial layer, especially surrounding ghost cells. Despite all cystic OTs in which MCs were found within the cystic epithelial lining, it is possible that there was a greater amount of antigen presenting molecules expressed by the MCs in the region involved in cell activation and recognition [10]. Therefore, considering the OTs as a group of lesions with heterogeneous morphology, one might think that the function of MCs could vary with the type of tumor and its morphological characteristics.
In conclusion, mast cells are probably one of the major components of the stromal scaffold in odontogenic tumors. Further studies should be carried out to try to establish a correlation between this cell population and other stromal components present in OTs, especially those involving angiogenesis.

\section{REFERENCES}

[1] Jing W, Xuan M, Lin Y, Wu L, Liu L, Zheng X, Tang W, et al. Odontogenic tumours: a retrospective study of 1642 cases in a Chinese population. Int J Oral Maxillofac Surg 2007; 36: 20-5.

https://doi.org/10.1016/j.ijom.2006.10.011

[2] Ledesma-Montes C, Mosqueda-Taylor A, Carlos-Bregni R, deLeon ER, Palma-Guzmán JM, Páez-Valencia C, MenesesGarcia A. Ameloblastomas: a regional Latin-American multicentric study. Oral Dis 2000; 13 : 303-7. https://doi.org/10.1111/j.1601-0825.2006.01284.x

[3] Caughey GH. Mast cell tryptases and chymases in inflammation and host defense. Immunol Rev 2007; 217: 141-54.

https://doi.org/10.1111/j.1600-065X.2007.00509.x

[4] Norrby K. Mast cells and angiogenesis. APMIS 2002; 110: 355-71.

https://doi.org/10.1034/j.1600-0463.2002.100501.x

[5] Conti P, Castellani ML, Kempuraj D, Salini V, Vecchiet J, Tete $\mathrm{S}$, Mastrangelo F, Perrella $\mathrm{F}$, et al. Role of mast cells in tumor growth. Ann Clin Lab Sci 2007; 37: 315-22.

[6] De Wever O, Mareel M. Role of tissue stroma in cancer cell invasion. J Pathol 2003; 200: 429-47. https://doi.org/10.1002/path.1398

[7] Valent $P$, Schernthaner $G H$, Sperr WR, Fritsch G, Agis $\mathrm{H}$,Willheim $M$, Buring $\mathrm{HJ}$, et al. Variable expression of activation-linked surface antigens on human mast cells in health and disease. Immunol Rev 2001; 179: 74-81. https://doi.org/10.1034/j.1600-065X.2001.790108.x

[8] Mangia A, Malfettone A, Rossi R, Paradiso A, Raniere G, Simone G, Restal L. Tissue remodeling in breast cancer: human mast cell tryptase as an initiator of myofibroblasts differentiation. Histopathology 2011; 58: 1096-106. https://doi.org/10.1111/j.1365-2559.2011.03842.x

[9] Maltby $S$, Khazaie K, McNagny KM. Mast cells in tumor growth: angiogenesis, tissue remodelling and immunemodulation. Biochim Biophys Acta 2009; 1796: 19-26. https://doi.org/10.1016/j.bbcan.2009.02.001

[10] Smith G, Smith AJ, Basu MK. Mast cells in human odontogenic cysts. J Oral Pathol Med 1989; 18: 274-8. https://doi.org/10.1111/j.1600-0714.1989.tb00396.x

[11] Li CY, Baek JY. Mastocytosis and fibrosis: role of cytokines. Int Arch Allergy Immunol. 2002; 127: 123-6. https://doi.org/10.1159/000048182

[12] Ribatti D, Ennas MG, Vacca A, Ferreli F, Nico B, Orru S, Sirigu $P$. Tumor vascularity and tryptase-positive mast cells correlate with a poor prognosis in melanoma. Eur $\mathrm{J}$ Clin Invest 2003; 33(5): 420-5. https://doi.org/10.1046/j.1365-2362.2003.01152.x 